\title{
ON AN IMPULSE INTERVAL GENERATING MECHANISM
}

\author{
M. TEN HOOPEN \\ Institute of Medical Physics TNO, UTRECHT/The Netherlands
}

This note is to be regarded as an addendum to an analysis of interval fluctuations of the sensory nerve impulse given in this journal by HAGIWARA (1954). In that paper observations, made on the intervals between successive impulses in afferent tonic fibres from muscle spindles of the toad upon stretching the attached muscle, were reported. It was found that the stationary sub-series of the impulse patterns could be considered as a random process. At modes of strong stimulation the mean interval duration was shorter and the dispersion smaller than for low strengths of the stretch and also after the sensory adaptation had come into play. With increase of the interval lengths not only the absolute value of the standard deviation but also its value relative to the mean became larger.

A model was developed that proved to be sufficient to express the shape of the interval histograms and the just-mentioned (non-linear) relationship between the mean and the spread of the durations between the impulses. It was based on the consideration that the initiation of a sensory nerve impulse is due to polarization of the terminal fibre, caused by a potential such as the generator potential of the muscle spindle. The moment an impulse in the nerve has been elicited the nerve is thrown in a state of depressed threshold. After a while it gradually recovers, becomes responsive again and the next impulse occurs. Furthermore, the interval distributions will be irregular if any amount of fluctuation exists in the process of initiation of the impulses.

More precisely, the properties of the model were defined as follows (cf. FIG. 1).

(1) The recovery process of the threshold $R(t)$ is expressed as $A \cdot \exp (c / t)$ when the preceding impulse has appeared at $t=0$. A denotes the rheobase of the fibre and $c$ is a constant in reference to this fibre.

(2) An impulse should occur when the following condition is fulfilled: $R(t)=S+X(t)$ or, in words, as soon as the momentary value of the stimulus intensity $S+X(t)$ is equal to the momentary threshold $R(t) . \quad X(t)$ is an additional random factor. It is assumed that $X(t)$ represents a random normal process, the mean being zero and the standard deviation $\sigma$, a constant which is not dependent on $S$ but only on the nerve fibre.

Received for publication August 15, 1964 


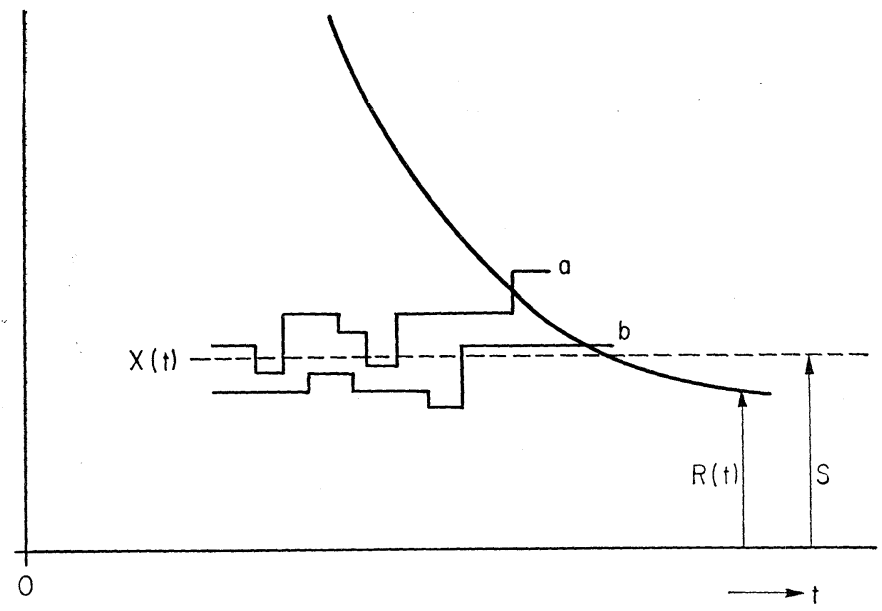

Fig. 1. Schematic representation of the course of recovery $R(t)$ in relation to the stimulus $S$ and two samples (a and b) of the random process $X(t)$ as a function of time $t$ after the last impulse has occurred at $t=0$.

The ultimate result was that the interval distribution could be written as:

$$
P(S, t)=\phi(S, t) \cdot \exp \left[-\int_{0}^{t} \phi(S, \tau) \cdot d \tau\right]
$$

with $\phi(S, t)=\int_{0=f(S, t)}^{\infty} \exp \left(-\frac{1}{2} z^{2}\right) \cdot d z / \sqrt{2 \pi}$ and $f(S, t)=[R(t)-S] / \sigma$.

\section{A MODIFIED MODEL}

Although expression (I), as a function of time continuous and with a maximum, furnished a good description for the observed histograms, it is nevertheless felt useful to point out that a random process as $X(t)$ has to be defined not only by the amplitude distribution of the momentary values but also by a property which characterizes the rapidity of their change with time. For it will be clear that the more frequently the function $S+X(t)$ " peaks" toward $R(t)$ the higher the chance that within a certain interval of time condition (2) is satisfied, all other parameters $(A, c, S$ and $\sigma$ ) having the same value. Consequently, the earlier in the mean an impulse appears after $t=0$ and the shorter the mean value between successive impulses. In the mathematical formalism this factor seems to be obscured and it is difficult to see how the rate of fluctuation of the process $X(t)$ is incorporated in expression (I).

It may be instructive to visualize the influence of this quantity exerted upon the mode of response of this threshold mechanism in Fig. 2. These 

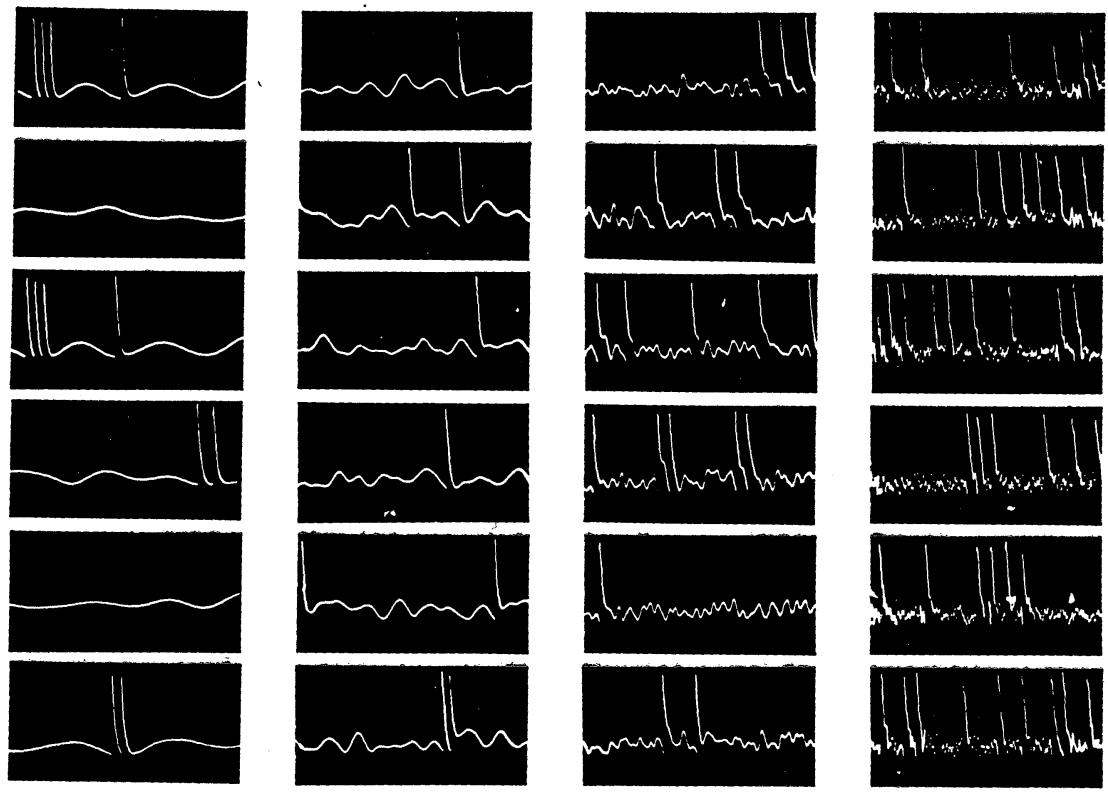

a

b

C

d

FIG. 2. Influence of the rate of fluctuation of $X(t)$ exerted upon the impulse pattern obtained from an electronically simulated process analogous to that of FIG. 1.

Recovery time course is equivalent to $R(t)=A$. $\exp (c / t)$ with $c=4 \mathrm{msec}$.

Length of each record equals sweep length $8.5 \mathrm{msec}$.

Large upward deflections indicate a response.

All parameters are the same except the upper high cut-off frequency of the noise spectrum which is from (a) through (d) equal to $300,1000,3000$ and 10,000 cps resp.

Note occasional "burst" activity in FIG. 2a.

records were obtained from an electronic model which simulated the conditions (1) and (2). Band-limited white noise was used for the random process $X(t)$. For technical reasons $X(t)$ was superimposed on $R(t)$ instead of on $S$. In view of the additivity of the functions involved and the symmetry of $X(t)$ this does not matter with respect to the moments of impulse ignition. In these oscillographic pictures the constant stimulus $S$ is not shown, merely $R(t)+X(t)$. The only difference between FIG. $2 a, b, c$ and $d$ is the value of the high cutoff frequency of the noise and increasing in this order from $300 \mathrm{cps}$ up to $10,000 \mathrm{cps}$, the lower limit being set at $20 \mathrm{cps}$. All other parameters, also the standard deviation $\sigma$, were the same throughout. It is seen from FIG. 2b, c and $d$ that the higher the rate of fluctuation the more responses are present per unit of time, an increase in the upper frequency limit by a factor 10 amounting to a decrease of the mean impulse interval by a factor 4 about. To FIG. 2a special attention will be paid later on. 
As these pictures are intended for illustrative purposes only it has not been tried to keep on the proper time scale in relation to the physiological prototype. The time constant of the recovery process was equivalent to $c=4$ msec. If the intervals and the constant $c$ are thought to be multiplied by a factor 25, thereby arriving at $c=100 \mathrm{msec}$ used by HAGIWARA, and correspondingly the noise cut-off frequency divided by this factor, arriving at 12,40 , 120 and $400 \mathrm{cps}$ respectively, we are in the range of those interval durations typically found for the muscle spindle discharges.

It must be remarked here that the following question must, on principle, be touched upon first before any model with a threshold mechanism can be elaborated appropriately at all, namely: what happens to the fluctuating function as soon as condition (2) is fulfilled and an impulse has appeared. Firstly, one may assume that after triggering an impulse the fluctuation starts with a new value, independent of the amplitude that has just been critical, is reset so to say. By definition then, there is no correlation between the durations of the subsequent intervals. Another possibility is that the fluctuation continues its course and is not affected by the reaction. In this case one must expect that sometimes short intervals cluster in a not-casual way. The extent to which this occurs depends on the system constants. For instance, the more slowly $X(t)$ varies with time and the more rapidly the recovery, the more frequently the situation is encountered that an accidental large excursion of the value of $S+X(t)$ gives rise to this burst activity. The electronic model was intentionally designed according to the directives of the second hypothesis and the burst phenomenon is clearly visible in FIG. 2a. If the noise frequency increases the moments of impulse ignition become less correlated.

HAGIWARA (1954) has found no detectable correlation between the duration of the successive intervals to be present. This is in favour of the first supposition but needs not exclude the second, if the rate of fluctuation of $X(t)$ is larger than the mean frequency of the discharge. For the problem at issue this last condition is indeed fulfilled as will be shown. Therefore, we are not forced to choose between these possibilities at the moment. In the interim the question suggests an interesting direction for future research.

In accordance with the foregoing we redefine $X(t)$ as a sequence of steps with normally distributed amplitudes (mean zero and standard deviation $\sigma$ ) while the moments of change are Poisson distributed (mean $\lambda$ ) as depictured in FIG. 1. This is not inconsistent with definition of $X(t)$ under point (2) in the Introduction. Even so much, they are probably identical in view of the assumption on which expression (I) was based, viz. that the probability of a reaction in a small time interval $(t, t+d t)$ is independent of the probability of a reaction in a similar preceding interval. It turns out that expression (I) remains valid provided we replace $\phi(S, t)$ by a quantity $\phi(S, t)$ which is derived in the Appendix. It is composed of two components which take in account 
the possibility of a vertical as well as of a horizontal intersection of $R(t)$ and the stepwise changing function $S+X(t)$.

We shall go a little into the subject by using expression (I) in the modified version to reexamine one of HAGIWARA's computed histograms, the one of his FIG. 5 that ranges from 50 to $110 \mathrm{msec}$ approximately (the time axis in that figure needs obviously to be multiplied by a factor 10 in order to match with his FIG. 4) and redrawn in FIG. 3. In doing so it is taken for granted that this curve corresponds with one of those experimentally found. As in the original communication $c$ was set equal to $100 \mathrm{msec}$ and, as to FIG. $3 \mathrm{~b}, A / \sigma=1$ whereas $S$ must have been about equal to $3 \sigma$. Parenthetically it can be remarked that the choice of the numerical values of these parameters

a

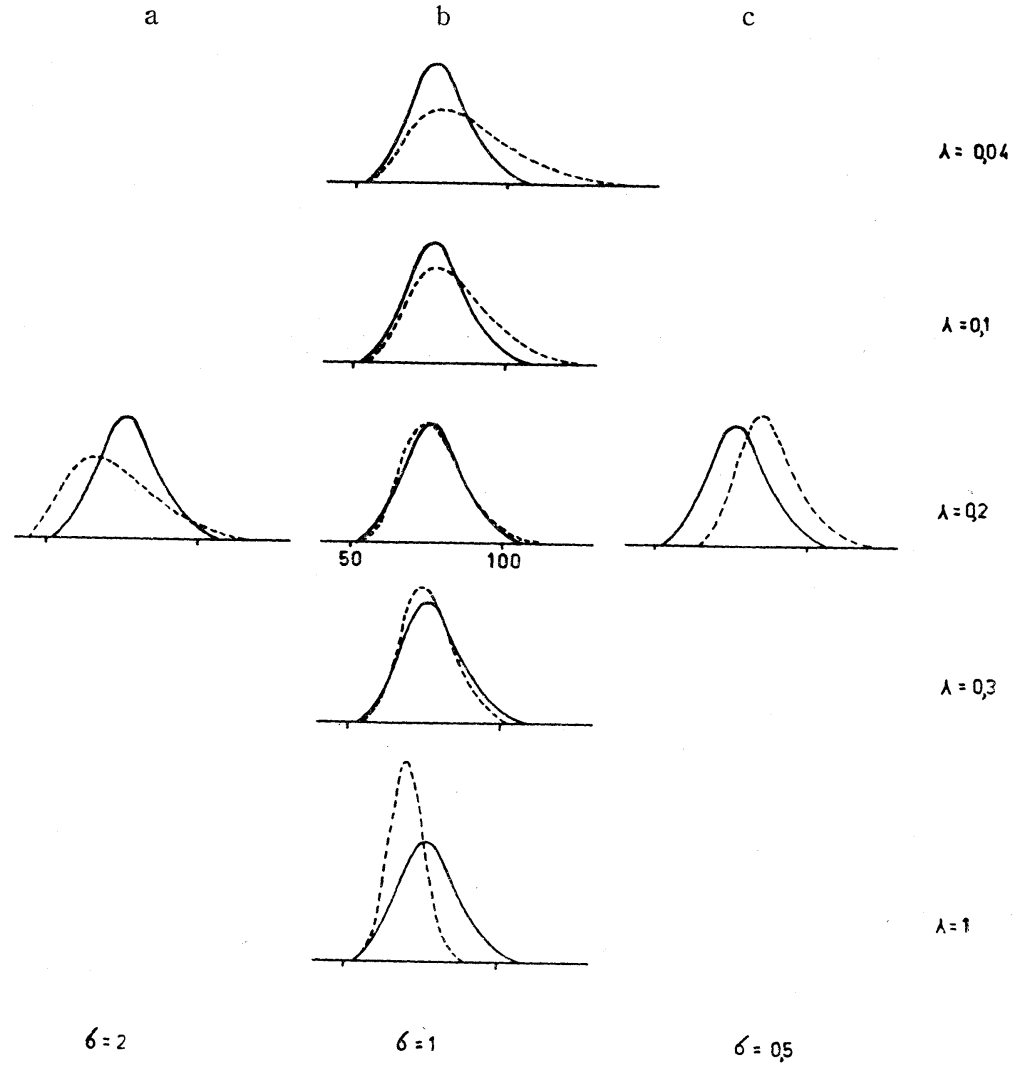

FIG. 3. Impulse interval histograms for different values of $\sigma$ and $\lambda$ (in $\mathrm{msec}^{-1}$ ).

Curves (…........) computed from formulae for $P(S, t)$.

Curves (—) adopted from one of the curves from HAGIWARA.

Time in msec.

$A=1, S=3, \sigma=2(\mathrm{a}), 1(\mathrm{~b})$ and $0.5(\mathrm{c})$. 
in expression (I) is rather restricted. So, if one wants to match analytically obtained and experimentally observed interval distributions, e. g. with respect to the beginning and the end (counting 1 resp. 99\% of the intervals) the value of $A / \sigma, S / \sigma$ and $c$ is dictated within a relatively narrow range of values.

A value of $\lambda=0.2$ per msec is seen to fit the given curve fairly good. Values of $\lambda=0.1$ and 0.3 per msec exhibit perceptible deviations. For other values of $\lambda$, as well as of $\sigma$ (FIG. 3a and c), the differences are more pronounced. The same order of magnitude for $\lambda$ has been found when other histograms were analysed (TEN HOOPEN, 1964). With reference to the remarks about the behaviour of the function $X(t)$ immediately after an impulse we can confirm now that a rate of change of 200 per sec of this function is indeed larger (more than ten times) than the mean discharge frequency, as was to be ascertained.

\section{DISCUSSION}

The main thesis in this note was that the definition of the irregular disturbances, which are thought to be the cause of the interval duration fluctuations of the sensory nerve impulse, should be extended in order to include the rate of fluctuation of said disturbances. This item has been referred to in the literature at a few places but scarcely in a well-elaborated way. Buller, Nicholls and STRÖM (1953) stated that the effect which fluctuations of the membrane potential have in accelerating or delaying the start of an impulse cannot be assessed without knowing the "frequency" as well as the r.m.s. (standard deviation) of the amplitudes of the fluctuations. VIERNSTEIN and Grossman (1961) paid more explicitly attention to this aspect in their investigation concerning the discharge patterns of single units in the cochlear and trigeminal nuclei, be it with the restriction that the rate of occurrence of the noisy disturbances was high with respect to the rate of firing of the cells.

In the present investigation a model has been described with a form of $X(t)$ that has also been useful to treat a strongly related phenomenon: fluctuation in nervous excitability (TEN Hoopen, Den Hertog and Reuver, 1963).

With only a minor formal modification the mathematical apparatus developed for that purpose can be applied to simulate interval fluctuations. This was indeed one of the reasons to comment upon the study of the muscle spindle discharges.

For the quantity that stands for the rate of fluctuation a value of about 200 per sec has been found. We are not in a position to identify this parameter and its value with a particular physiological process. One possibility is that it might reflect the rate of impinging, by spatio-temporal summation, of many subliminal events from the muscle spindle receptors, in analogy with 
the well-known motor endplate and synaptic miniature potentials, thereby causing the membrane potential of the nerve fibre at the point of impulse ignition to very irregular round some equilibrium state.

One objection of HAGIWARA to his model was that the estimated value of $\sigma$ is rather large relative to the rheobase of the nerve fibre A. This selfcriticism is perhaps not that serious in view of recent findings of VERVEEN (1963) who inferred, from experiments on nerve fibres stimulated with electric currents, an empirical relation between $\sigma / A$ and the fibre diameter for diameters ranging from a few to a few hundred microns. If this may be extrapolated to smaller diameters one finds $\sigma / A=1$ for a diameter of 0.03 microns. This value appeals to some extent to the conclusions of a histological study by BARKER (1961), who reported the presence of a number of extremely fine nerve fibres in spindles, which innervate the intrafusal muscle fibres in the equatorial region.

They measure 0.5 micron or less in diameter, branch extensively and end as free epilemmal terminals in the area of sensory innervation. By this branching process the diameter may well be cut down several times and thus approaching the above mentioned value. Furthermore, according to BARKER, the most probable interpretation appears to be that they are sympathetic fibres such as HUNT (1960) has shown cause significant changes in the threshold of spindle receptors to applied stretch.

The rheobase being of the same order as the noise intensity, and given the used rate of recovery $(c)$ and the ascertained value of $\lambda$, has as a consequence that in the absence of an intentional stimulus spontaneous discharges should be present. For $S=0$ one finds a mean discharge interval of $250 \mathrm{msec}$. This seems to be in harmony with a mean interval of nearly $200 \mathrm{msec}$ found for a stretch strength of only $2 \mathrm{mg}$ and after adaptation. This situation is probably near the border of no stimulation, were it alone in view of the maximum applied stretch of $300 \mathrm{mg}$.

However, as the exact relations between the stimulus $S$ used in expression (I) of the model and the proper stimulus due to stretch is not known-another threshold mechanism in the transducer mechanism might well be present-it does not seem opportune to go into further possible inductions at the moment.

\section{SUMMARY}

1. Some of the data obtained by HAGIWARA (1954) on the interval fluctuations of the sensory nerve impulse have been reanalysed with a modified model.

2. The modification consisted essentially of the introduction of a factor characterizing the rate of fluctuation of a random process that is thought to be super-imposed on the threshold of the impulse triggering mechanism.

3. The mean rate of change in amplitude of the step-wise defined random 
process was found to be in the order of a few hundred per second.

4. Some of the implications of the model: the time course of the random function immediately after an impulse, the large value of the noise intensity relative to the rheobase and, as a consequence, the predicted existence of spontaneous discharge, were discussed.

\section{REFERENCES}

1) Barker, D. and Cope, M. Symposium on Muscle Receptors, D. Barker, Ed., Hong Kong, 1961.

2) Buller, L. J., Nicholls, J. G. And Ström, G. J. Physiol. 122: 409, 1953.

3) Hagiwara, S. Japan J. Physiol. $4: 234,1954$.

4) Hunt, C. C. J. Physiol. 151: 332, 1960.

5) Ten Hoopen, M., Den Hertog, A. and Reuver, H. A. Kybernetik 2: 1, 1963.

6) Ten Hoopen, M. Unpublished results, 1964.

7) Verveen, A. A. Acta Morph. Neerland.-Scand. $5:$ 79, 1963.

8) Viernstein, L. J. and Grossman, R. G. Information Theory, C. Cherry, Ed., London, 1961.

\section{APPENDIX}

In general one can define $\psi(S, t) . \Delta t=$ the conditional probability of a response in the time interval $(t, t+\Delta t)$ provided no response has yet occurred in the interval $(0, t)$. In case of the random step train the probability $\psi(S, t)$. $\Delta t$ is composed of the following two mutually excluding probabilities: (a) that $S+X(t)$ jumps upward, in the nomenclature of FIG. 1, during an interval $\Delta t$ and intersects $R(t)$ vertically, and (b) that $S+X(t)$ remains constant but $R(t)$ becomes that low in the interval $\Delta t$ so as to become intersected horizontally. After elaboration and passing to the limit for $\Delta t \rightarrow 0$ one obtains (Ten Hoopen, Den Hertog and Reuver, 1963)

$$
\psi(S, t)=\lambda \phi(S, t)+\frac{d}{d t}[\phi(S, t)] /[1-\phi(S, t)] .
$$

Then by definition the probability that a response occurs for the first time after $t=0$ in $(t, t+\Delta t)$ is given by:

$$
\begin{aligned}
P(S, t) \cdot \Delta t & =\left[1-\psi\left(S, t_{0}\right) \cdot \Delta t\right] \cdot\left[1-\psi\left(S, t_{1}\right) \cdot \Delta t\right] \\
\ldots & {\left[1-\psi\left(S, t_{n-1}\right) \cdot \Delta t\right] \cdot\left[\psi\left(S, t_{n}\right) \cdot \Delta t\right] }
\end{aligned}
$$

with $t_{0}=0, t_{1}=\Delta t, \ldots t_{k}=k \cdot \Delta t, \ldots t_{n}=t$.

After passing to the limit it follows for $\Delta t \rightarrow 0$

$$
P(S, t)=\psi(S, t) \cdot \exp \left[-\int_{0}^{t} \psi(S, \tau) \cdot d \tau\right] .
$$

